\title{
Deep learning and evolutionary intelligence with fusion-based feature extraction for detection of COVID-19 from chest X-ray images
}

\author{
K. Shankar ${ }^{1}$ (1) $\cdot$ Eswaran Perumal ${ }^{1} \cdot$ Prayag Tiwari $^{2} \cdot$ Mohammad Shorfuzzaman $^{3} \cdot$ Deepak Gupta $^{4}$
}

Received: 31 January 2021 / Accepted: 17 April 2021 / Published online: 27 May 2021

(c) The Author(s), under exclusive licence to Springer-Verlag GmbH Germany, part of Springer Nature 2021

\begin{abstract}
In recent times, COVID-19 infection gets increased exponentially with the existence of a restricted number of rapid testing kits. Several studies have reported the COVID-19 diagnosis model from chest X-ray images. But the diagnosis of COVID-19 patients from chest X-ray images is a tedious process as the bilateral modifications are considered an ill-posed problem. This paper presents a new metaheuristic-based fusion model for COVID-19 diagnosis using chest X-ray images. The proposed model comprises different preprocessing, feature extraction, and classification processes. Initially, the Weiner filtering (WF) technique is used for the preprocessing of images. Then, the fusion-based feature extraction process takes place by the incorporation of gray level co-occurrence matrix (GLCM), gray level run length matrix (GLRM), and local binary patterns (LBP). Afterward, the salp swarm algorithm (SSA) selected the optimal feature subset. Finally, an artificial neural network (ANN) is applied as a classification process to classify infected and healthy patients. The proposed model's performance has been assessed using the Chest X-ray image dataset, and the results are examined under diverse aspects. The obtained results confirmed the presented model's superior performance over the state of art methods.
\end{abstract}

Keywords Fusion model $\cdot$ Metaheuristic $\cdot$ COVID-19 $\cdot$ Feature extraction $\cdot$ Classification

\section{Introduction}

K. Shankar

drkshankar@ieee.org

Eswaran Perumal

eswaran@alagappauniversity.ac.in

Prayag Tiwari

prayag.tiwari@ieee.org

Mohammad Shorfuzzaman

m.shorf@tu.edu.sa

Deepak Gupta

deepakgupta@mait.ac.in

1 Department of Computer Applications, Alagappa University, Karaikudi, India

2 Department of Computer Science, Aalto University, Espoo, Finland

3 Department of Computer Science, College of Computers and Information Technology, Taif University, P.O. Box 11099, Taif 21944, Saudi Arabia

4 Department of Computer Science and Engineering, Maharaja Agrasen Institute of Technology, Delhi, India
Nowadays, COVID-19 disease has been a fatal and dangerous one globally [1]. The novel corona-virus initially affects the throat and gradually moves in humans' respiratory tract. It completely spreads over the lungs, leading to high fever, throat pain, and many other consequences named 'Pneumonia'. The architecture of COVID-19 is depicted in Fig. 1 [2]. If the novel coronavirus enters into the body, lungs are occupied with fluid, and get infected, which becomes patches named 'Ground-Glass Opacity (GGO)'. It is highly tough to predict the diseases due to the limited healthcare facilities and clinics [3-10]. It is essential to discover an effective remedy for the disease-analyzing process.

Even under a complicated situation, massive efforts have been taken to find an efficient approach to predict the existence of COVID-19. The better way to control the disease is social distancing, quarantine, and using sanitizers for hands and surrounding places, which many countries have followed. Unfortunately, the lockdown affects a country's GDP, and it also affected the people psychologically and physically. As the COVID-19 is an air-based disease, it can easily be spread by nature ailments like group interactions, 
Fig. 1 Structure of Coronavirus (COVID-19)

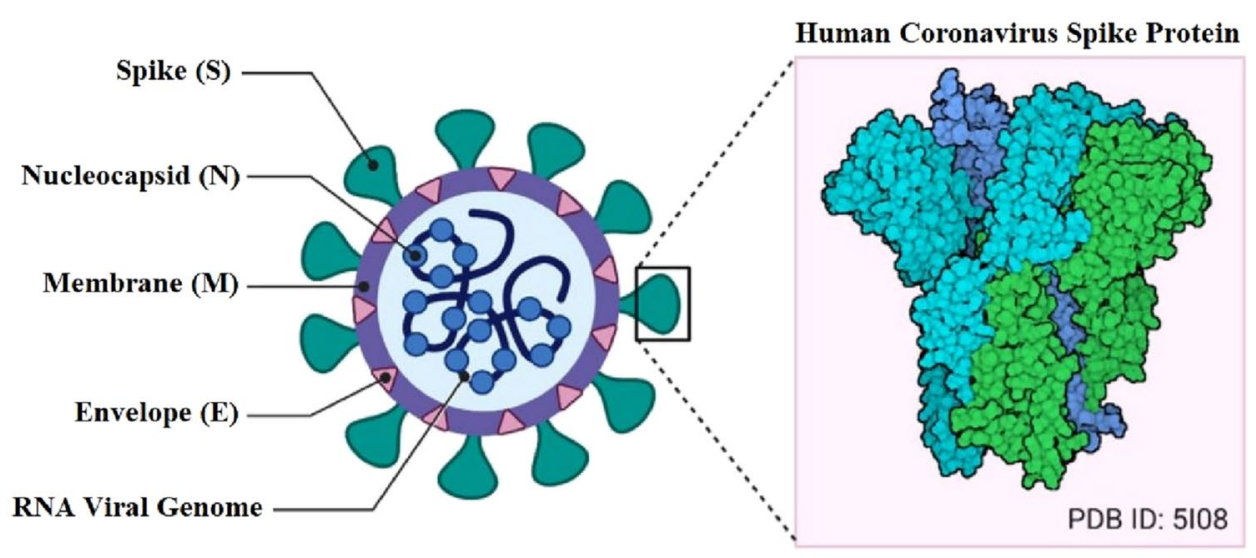

crowds, and places like malls and theaters are some of the sources in the disease spreading. Even though the measures are insisted regularly, peoples are affected by COVID-19. The highly affected countries such as the USA, Italy, and Spain reached a higher death count than China. A scientist defined that "This virus is as economically contagious as it is medically contagious". Hence, it is compulsory to develop a healthcare prediction model using artificial intelligence (AI), which is applicable for predicting the cases rapidly and precisely to eliminate a higher mortality rate.

Here, Deep Learning (DL) method is applied because of the massive benefits. It has changed many applications [11], specifically in the clinical sector, image database like retinal image, chest X-ray, and brain MRI, which offers challenging outcomes with better accuracy. Previously, an X-ray is a scanning machine that provides cheaper and rapid outcomes while screening internal organs and bones. Understanding diverse X-ray images are highly carried out physically by professional radiologists. It is more helpful for developing countries where X-ray service is accessible; however, more experts are not available. Also, DL aims to deploy a deep neural network (DNN) termed as 'nCOVnet', which examines the lung's X-ray image and predicts corona's presence. Initially, computer-aided clinical predictive methods were applied to diagnose different kinds of diseases. Using these approaches, medical professionals comprehend and apply clinical findings as well. The identical studies regarding clinical detection for COVID-19 [12, 13] have restricted characteristics.

This paper devises an effective and intelligent healthcare system using a fusion model for COVID-19 diagnosis. The presented technique comprises different preprocessing, feature extraction, and classification processes. Preprocessing of images is done using the WF technique. Subsequently, the fusion-based feature extraction process is carried out by incorporating GLCM, GLRM, and LBP. Afterward, the optimal feature subset is selected by SSA. Lastly, ANN is applied as a classification process to classify the infected and healthy persons. The ANN is preferred due to the following characteristics: it requires less formal statistical training, the ability to implicitly detect complex nonlinear relationships between dependent and independent variables, and the ability to detect all possible interactions between predictor variables. The proposed technique has been used with the Chest Xray image database's help, and the results are examined under diverse dimensions.

\section{Related works}

Machine learning (ML) models are applied to detect coronavirus's disease level. Various ML algorithms such as logistic regression (LR), $k$-nearest neighborhood (KNN), two diverse decision tree (DT), random forest (RF) as well as support vector machines (SVM) classification models can be employed for COVID-19 diagnosis. The working function of these classifiers is estimated with accuracy values. The SVM classifier provides optimal accuracy. Besides, [14] have used the ML classification method for detecting COVID-19. Medical information was collected, and clinical findings are assumed as well as the performance of the ML classifier is determined with respect to different measures. In Ref. [14], 5 distinct classifiers have been utilized, such as SVM, RF, neural networks (NN), LR, and gradient boosted trees (GBT). The maximum AUC values can be attained using SVM and RF classification methodologies. In Ref. [15], a clinical predictive method for COVID-19 has been presented using the data accumulated from the hospitals. Several research works used diverse ML models like RF, NN, LR, SVM, GBT and assessed the classification models' function using sensitivity, specificity, and AUC scores. In this metric, an optimal function can be achieved using XGB with the best AUC score.

Researchers have developed a diverse range of methods to analyze medical data to enhance the medical sciences. For this purpose, Several classification approaches were used 
effectively to obtain related features from image databases. The COVID19 disease affects the human lungs, which can be identified by examining the lung X-ray. A successful CNN approach has been used with a convolutional neural network $(\mathrm{CNN})$ to predict the Pneumonia case from chest X-ray [16-20]. The training process take place on a set of images offering the front view of lung X-ray images [21]. An automatic COVID-19 detection model was proposed by Shorfuzzaman and Hossain et al. [22], which uses Simese network classifiers with a CNN encoder for the prediction with maximum accuracy.

Singh and Gupta [23] utilized a DL model with rectified linear unit (ReLU) activation function for the detection of lung cancer. Esteva et al. [24] concentrated on the categorization of skin lesions. The model undergoes sampling and is divided into two classes: general cancer and malicious cancer. Liu et al. [25] developed a model for classifying the chest's tuberculosis. The given X-ray image database is inappropriate. Thus, it lacks in assuming region-level data at data preprocessing.

Butt et al. [26] employed ResNet-23 and conventional ResNet-18, which is applicable to obtain better accuracy by training the CT Scan images. Therefore, the maximum time is required, which is not accessible in many clinics. Fanelli and Piazza [27] applied a commonly accessible database collected to predict the movements of COVID-19 under various cases. Using mean-field kinetics of pandemic spreading, it detects the existence of COVID-19, and lockdown imposed by the government has changed the lifestyle of many peoples. Apostolopoulos and Mpesiana [28] used artificial intelligence (AI) with diverse transfer learning and reached better outcomes using VGG19 with optimal accuracy. The applied database was collected from Cohen's GitHub, but it eliminated the data leakage, which happens because of the maximum images about a similar patient.

Li et al. [29] employed AI with 3D DL approach to predict COVID-19 patients on a database with higher CT Scans of many patients. An automatic method was presented in [30] under DL and AI applications, especially LSTM networks [6], to detect the trends and feasible cessation time of COVID-19 in diverse countries. The approach utilized in time-series data for predicting Canada's future virus trends and various infection clusters have existed recently. Though several models are available in the literature, there is still a need to design a fusion-based COVID-19 diagnosis model to achieve an improved detection rate.

\section{The proposed method}

The workflow involved in the presented methodology is shown in Fig. 2. As depicted, the figure indicates that the proposed model involves different processes, namely preprocessing, feature extraction, and classification.

\subsection{Preprocessing using Weiner filtering method}

Noise elimination is defined as an image preprocessing model used to enhance the image's properties interrupted by noise. In particular, wiener filtering is applied in which denoising is performed according to the image's noise content. It is used due to the following benefits: it exploits signals effectively, controls the output error, and is easy to implement. Consider the corrupted image, which is calculated by, $\widehat{I}(x, y)$, the noise variance of the complete image has been showcased by, $\sigma_{y}^{2}$, the local mean is provided by, $\widehat{\mu_{L}}$ over a pixel window as well as local variance from a window is depicted by, $\hat{\sigma}_{y}^{2}$. Followed by, a feasible path in denoising an image is represented as:

$\widehat{\widehat{I}}=\widehat{I}(x, y)-\frac{\sigma_{y}^{2}}{\hat{\sigma}_{y}^{2}}\left(\widehat{I}(x, y)-\widehat{\mu_{L}}\right)$.

Then, if the noise variance over an image is 0 ,

$\sigma_{y}^{2}=0=>\widehat{I}=\widehat{I}(x, y)$.

When global noise variance is minimal, and local variance is maximum than global variance becomes 1 ; so that, if

$\widehat{\sigma}_{y}^{2^{\prime \prime}} \sigma_{y}^{2}$, then $\widehat{\widehat{I}}=\hat{I}(x, y)$.

The maximum local variance shows the existence of an edge from the image window. In case of the same local and global variances, the function can be expressed as:

$\widehat{\hat{I}}=\widehat{\mu_{L}} \operatorname{as} \widehat{\sigma}_{y}^{2} \approx \sigma_{y}^{2}$.

The above analogies simplify a mean where output is a mean value of a window over a pixel without any anomalies. It is an inherent functionality of WF. The filter applies window size as input and determines the remaining objectives [31]. The attained simulation outcome projected the work in noise elimination from Chest X-ray images using WF. The key objective is to learn the impact of WF noise elimination on edges.

\subsection{Fusion of feature extraction}

In this section, the fusion of feature extraction takes place using the hybridization of GLCM, GLRM and LBP features. Besides, the optimal set of features is then extracted using SSA. 
Fig. 2 Flowchart of Proposed FM-ANN model

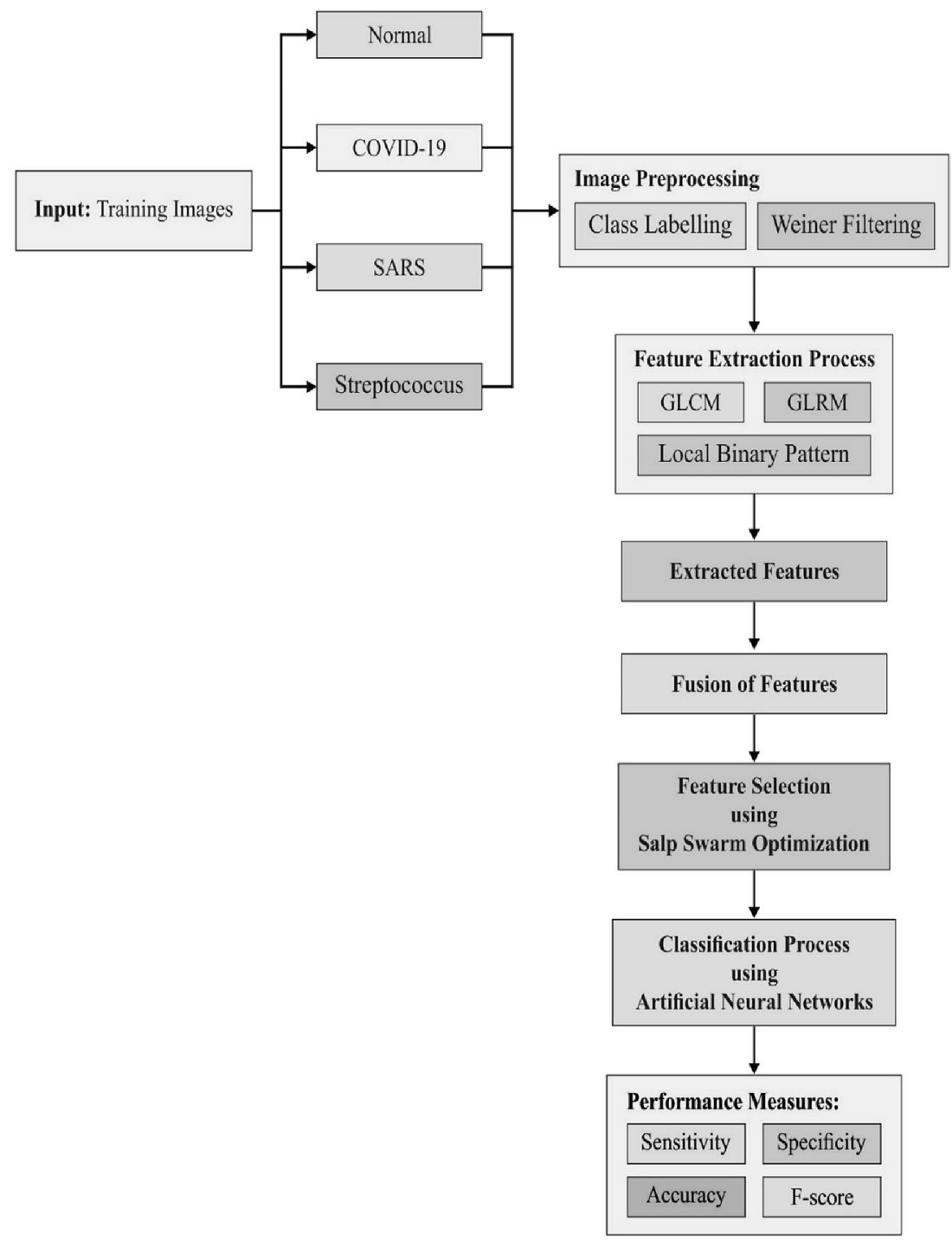

\subsubsection{GLCM features}

The GLCM is defined as a statistics-based model that assumes the spatial connection over pixel [32]. It is a 2D histogram that determines the significance of pixel divided by a specific distance $d_{\text {occ }}$ that exists in the image. Suppose $I(i, j)$ be the image of size $N \times M$, with $L$ gray levels, $I\left(i_{1}, j_{1}\right)$ and $I\left(i_{2}, j_{2}\right)$ be 2 pixels with gray level intensity $x_{1}$ and $x_{2}$, correspondingly. While taking $\Delta i=i_{2}-i_{1}$ in $i$ direction and $\Delta j=j_{2}-j_{1}$ in $j$ direction, the connecting line has direction $\theta$ that is same as $\arctan \left(\frac{\Delta j}{\Delta i}\right)$. The generalized co-occurrence matrix $C_{\theta, d}$ is depicted as:

$C_{\theta, d}\left(x_{1}, x_{2}\right)=\left(\operatorname{Num}\left\{\left(\left(i_{1}, j_{1}\right),\left(i_{2}, j_{2}\right)\right) \varepsilon(N \times M) \times(N \times M) / A\right\}\right) / K$.

In this model, $A$ is defined as a provided condition, like $d_{\mathrm{occ}} \sin (\theta), \Delta j=d_{\mathrm{occ}} \cos (\theta)$. Num shows the value of components in co-occurrence matrix and $K$ implies the overall count of pixels. Generally, $d_{\mathrm{occ}}=1,2$ and $\theta=0^{\circ}, 45^{\circ}, 90^{\circ}, 135^{\circ}$ have been applied for estimations. The 5 diverse texture features are described under the application of co-occurrence matrix as provided:

Energy $=\sum_{i} \sum_{j} C_{\theta, d}^{2}(i, j)$

Variance $=\frac{1}{N \times M} \sum_{i} \sum_{j}\left(C_{\theta, d}(i, j)-m\right.$

Entropy $=-\sum_{i} \sum_{j} C_{\theta, d}(i, j) \log _{2}\left(C_{\theta, d}(i, j)\right)$

Contrast $=\sum_{i} \sum_{j}(i-j)^{2} C_{\theta, d}(i, j)$ 
Homogeneity $=\sum_{i} \sum_{j} \frac{C_{\theta, d}(i, j)}{1+|i-j|}$,

where $m$ implies mean value of $c o^{-o c c u r r e n c e ~ m a t r i x ~} C_{\theta, d}$

\subsubsection{GLRM features}

The GLRLM provides the dimension of homogeneous runs for all gray levels. It is determined for 4 various directions, and each one has 11 texture indices obtained from the matrices. The component $(i, j)$ of GLRLM links homogeneous runs of $j$ pixels with intensity $i$ from an image named as $\operatorname{GLRLM}(i, j)$. Assuming the GLRM matrix with 11 features as mentioned above is attained. For all images, 44 features (11 features $\times 4$ directions ) have been accomplished [33].

Where $Q(\mathrm{i}, \mathrm{j})$ implies a GLRM matrix, $i$ shows a graylevel measure, $j$ depicts run length, and $S$ denotes the total of every value in GLRM matrix.

Shortrun $=\sum_{i} \sum_{j}\left(Q(i, j) / j^{2}\right) / S$

Longrun $\left.=\sum_{i} \sum_{j} 0^{2} Q(i, j)\right) / S$

Graylevelnon-uniformity $=\sum_{i},\left(\sum_{j} Q(i, j)\right)^{2} / S$

Runlengthnon-uniformity $=\sum_{j},\left(\sum_{i} Q(i, j)\right)^{2} / S$

Runratio $=\sum_{i} \sum_{j} S / j Q(i, j)$

Low gray level run $=\sum_{i} \sum_{j} Q(i, j) / S i^{2}$

$H$ igh gray level run $=\sum_{i} \sum_{j} i^{2} Q(i, j) / S$

Short run low gray level $=\sum_{i} \sum_{j} Q(i, j) / S j^{2} i^{2}$

Short run high gray level $=\sum_{i} \sum_{j} i^{2} Q(i, j) / S j^{2}$

Long run low gray level $=\sum_{i} \sum_{j} j^{2} Q(i, j) / S i^{2}$

\subsubsection{LBP features}

The LBP is defined as a statistical model applied in image processing that accomplishes efficient features from images [34]. It is highly used in the computer vision domain. This operator develops a binary value $S\left(f_{P}-f_{c}\right)$ for all pixels under the comparison of the center pixel $(f)$ as well as surrounding pixels $f_{P}=(P=0,1, \ldots, 7)$ around $3 \times 3$. LBP measures are accomplished by binarizing the variations among the neighbors of pixels in all images using a function (Eq. (21)).

$\operatorname{LBP}_{P, R}\left(x_{C}\right)=\sum_{p=0}^{P-1} \mu\left(x_{p}-x_{c}\right) 2^{p}, \mu(y)=\left\{\begin{array}{l}1, y \geq 0 \\ 0, \mathrm{y}<0\end{array}\right.$

In Eq. 21, $R$ denotes the radius and species of distance from neighboring pixels to middle pixel, while $P$ refers to the count of nearby pixel values. Uniform patterns are defined as binary LBP code of $0-1$ or 1-0, and the count of transition is 2 . Uniform patterns represent simple textures like spot, edge, and corner. It is also composed of $(P-1) P+2$ uniform patterns.

\subsection{Optimal feature selection using SSA}

\subsubsection{SSA}

SSA is defined as a random population-relied method recommended by [35]. It is used for accelerating the swarming process of salps while foraging in the ocean. In the deeper ocean, the salps model, a swarm, named as salp chain. In the SSA approach, the dominant is a salp in front of the chain, and balance salps are termed, followers. The salps location can be saved in a 2D matrix named as $z$. Moreover, the food source is referred to as $P$ in search space as the swarm destination. The numerical approach for SSA is provided in the following: the dominant salp will modify the location under the application of given function:

$z_{n}^{1}=\left\{\begin{array}{l}P_{n}+r_{1}\left(\left(u_{n}-l_{n}\right) r_{2}+l_{n}\right) r_{3} \geq 0 \\ P_{n}-r_{1}\left(\left(u_{n}-l_{n}\right) r_{2}+l_{n}\right) r_{3}<0\end{array}\right.$

$r_{1}=2 e^{-}\left(-\frac{4 a}{A}\right)^{2}$

The coefficient $r_{1}$ is a necessary attribute in SSA as it offers better management among exploration and exploitation phases. To modify the position of followers, given functions are applied:

$z_{n}^{m}=\frac{1}{2} c e^{2}+v_{0} e$,

where $m \geq 2, c=\frac{v_{\text {rinal }}}{v_{0}}$ where $v=\frac{z-z_{0}}{e}$. Due to the time in optimization, the crisis among iterations are 1 , and assuming $v_{0}=0$, which has been determined by:

$z_{n}^{\mathfrak{m}}=\frac{1}{2}\left(z_{n}^{\mathfrak{m}}+z_{n}^{\mathfrak{m}-1}\right)$. 
A brief stepwise definition of this approach is given in the following:

1. Upload the parameters of SSA like count of salps $(S)$, count of iteration $(A)$, best salp position $\left(Z^{*}\right)$ and optimal fitness value $\left(f\left(Z^{*}\right)\right)$.

2. Upload a population of $S$ salp's position arbitrarily.

3. Estimate the fitness of all salps.

4. Fix count of iteration to 0 .

5. Upgrade $\mathrm{r} 1$.

6. For all salps,

(a) If $m==1$, upgrade the place of leading salp by Eq. (22).

(b) Else, upgrade the place of follower salp by Eq. (25).

(c) Determine the fitness of all salps.

(d) Upgrade $Z^{*}$ when the attained solution is better than the existing solution.

7. Increase a to 1 .

8. Follow Steps $5-7$ till $a=A$ is met.

9. Provide the optimal solution $Z^{*}$ and fitness value $f\left(Z^{*}\right)$.

\subsubsection{Solution representation}

In feature selection (FS) issues, each solution is restricted from $[0,1]$ values. For the SSA approach, which is applied with FS problem, a binary version must be deployed. Here, a solution is described as a $1 \mathrm{D}$ vector, where the length of a vector depends upon the features in the actual dataset [36]. The cell in a vector contains the measure of " 1 " or " 0 ". Value " 1 " represents the corresponding feature as selected; else, the measure is depicted as " 0 ". Equation (26) is applied for mapping continuous measures into binary ones.

$Z_{m n}=\left\{\begin{array}{l}1 \text { if } X_{\mathfrak{m} n}>0.5 \\ \text { 0otherwise }\end{array}\right.$

where $Z_{m n}$ represents the discrete form of solution vector $X$ and $X_{m n}$ Implies continuous locations of search agent $m$ at dimension $n$.

\subsubsection{Fitness function}

The FS is labeled as a multi-objective optimization issue in which two conflicting objectives should be obtained; minimum selected features and higher classification accuracy. The best solution can be identified where a solution with a lower count of selected features as well as maximum classification accuracy. For effective management of chosen features as well as classification accuracy, the fitness function in Eq. (27) has been applied in ISSA, and these models apply to the search agents.
Fitness $=\rho \operatorname{Err}(D)+\phi \frac{|F|}{|T|}$,

where $\operatorname{Err}(\mathrm{D})$ demonstrates classifier error rate, $\rho$ and $\phi$ are constants for controlling classification accuracy and feature limitation, $|F|$ refers to the size of discovered feature subset, $|T|$ signifies overall count of features. $\rho$ in $[0,1]$ and $\phi=(1-\rho)$, thus, $\rho=0.9$. Fig. 3 shows the flowchart of SSA for FS.

\subsection{Classification using ANN}

The ANN structure of multilayer perceptron (MLP) is composed of specification of several layers, the type of activation function for all units, and weights of connections among various units, and has to be implemented for developing neural structure [37, 38]. It offers a supervised feedback ANN structure in three layers: the input layer shows limited rule base measures as shown in Fig. 4.

The structure of ANN involves input, hidden, and output layers. The first layer indicates the attributes of the data. The second hidden layer defines the attributes that are linearly unseparable and output layer offers the required outcome.

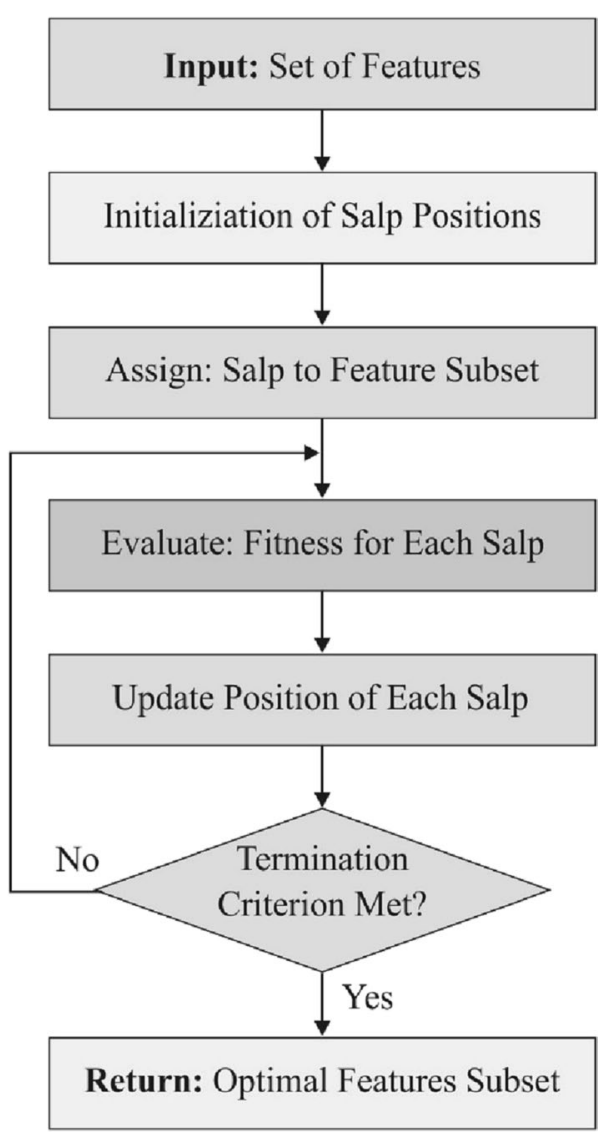

Fig. 3 SSA for feature selection 
The threshold node is moreover appended in the input layer specifying the weight function. The resulting function can be represented as follows:

$p_{j}=\sum_{i=1}^{n} w_{j, i} x_{i}+\theta_{j}, m_{j}=f_{j}\left(p_{j}\right)$

where $p_{j}$ is the linear amalgamation of input $x_{1}, x_{2}, \ldots, x_{n}$, and the threshold $\theta_{j}, w_{j, i}$ is the connection weights among input $x_{i}$ and the neuron $j, f_{j}$ is the $j$ th neuron's activation function, and $m_{j}$ is the output. A sigmoid function is an ordinary choice of the activation function as given below.

$f(t)=\frac{1}{\mathrm{e}^{-t}}$

For training MLP, the backpropagation learning concept has been employed, which is a gradient descent model for weight adaptation. Every weight vector $(w)$ is initialized to an arbitrary value. The pattern is computed layer by layer till the output layer offers a response. ANN contains the capability to resolve the issues in tedious systems like image classification, and it helps to classify different classes of COVID-19.

\section{Performance validation}

In this section, the presented technique has been validated using the Chest X-ray dataset [39], which encompasses 27 images under normal class, 220 images under SARS class, and 17 images under Streptococcus. For experimentation, a tenfold cross-validation process is employed. The proposed model is implemented using Intel i5, 8th generation PC with 16 GB RAM, MSI L370 Apro, Nividia 1050 Ti4 GB. For experimentation, Python 3.6.5 is used along with pandas, sklearn, Keras, Matplotlib, TensorFlow, opencv, Pillow, seaborn, and pycm. The parameters involved are batch size: 128 , learning rate: 0.001 , epoch count: 500 , and momentum: 0.2 . Figures 5 and 6 illustrate some test images from binary and multiple classes of COVID-19 chest X-ray images. The sample screenshots attained at the time of the execution is given in Appendix.

Table 1 and Fig. 7 illustrate the FM-ANN model's results for the classification of binary class in terms of different aspects. Under the $\mathrm{CV}-1$, the proposed FM-ANN model has resulted in a higher sensitivity of $94.38 \%$, specificity of $95.30 \%$, accuracy of $94.29 \%$, and an $F$ score of $95.09 \%$, respectively. Likewise, under the $\mathrm{CV}-2$, the presented FM-ANN approach has provided maximum sensitivity of $95.17 \%$, specificity of $95.43 \%$, accuracy of $95.64 \%$, and an $F$ score of $94.85 \%$ correspondingly. Followed by, under the CV-3, the projected FM-ANN method has provided maximum sensitivity of $94.87 \%$, specificity of $96.76 \%$, accuracy of $96.40 \%$, and an $F$ score of $95.32 \%$, respectively. Moreover, under the CV-4, the developed FM-ANN technology has provided high sensitivity of $94.32 \%$, specificity of $95.89 \%$, accuracy of $95.88 \%$, and an $F$ score of $96.83 \%$ correspondingly. Moreover, under the CV-5, the proposed FM-ANN approach has obtained maximum sensitivity of $96.74 \%$, specificity of $95.30 \%$, accuracy of $94.29 \%$, and an $F$ score of $95.09 \%$, respectively.

Similarly, the presented FM-ANN approach's CV average analysis has provided a sensitivity of $95.10 \%$, specificity of $95.97 \%$, accuracy of $95.91 \%$, and an $F$ score of $95.29 \%$ correspondingly.

Table 2 and Fig. 8 demonstrate the FM-ANN approach's results for Multi-class classification using diverse aspects. Under the CV-1, the applied FM-ANN technology has

Fig. 4 Structure of ANN

Input Layer

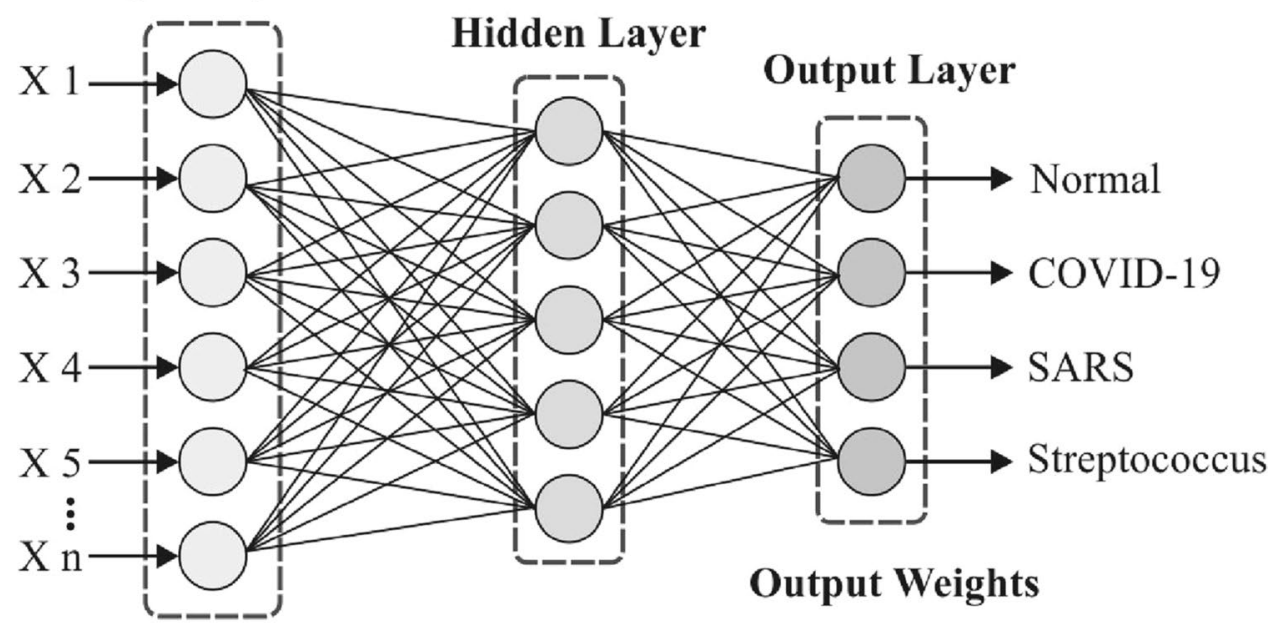

Input Weights 

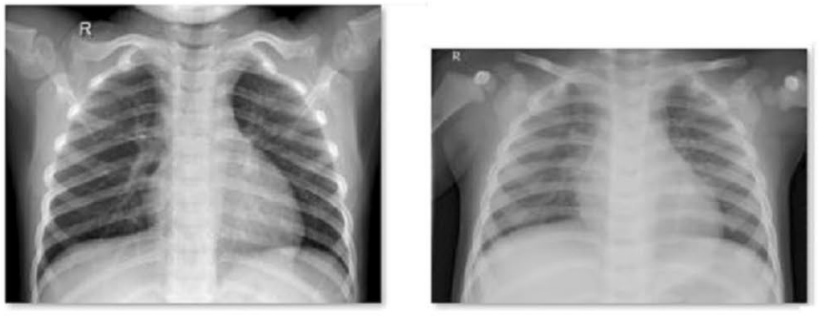

(a)

Fig. 5 Sample binary class images
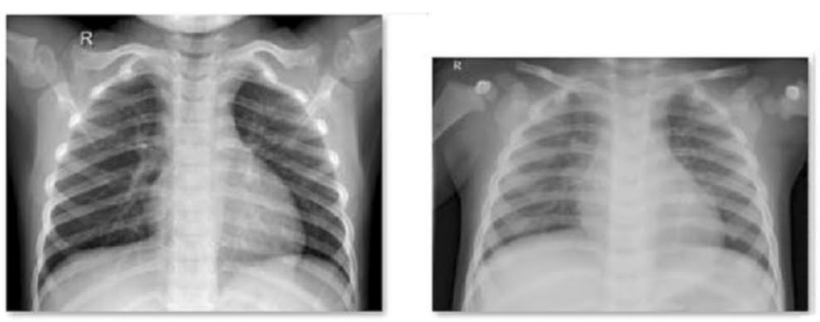

(a)
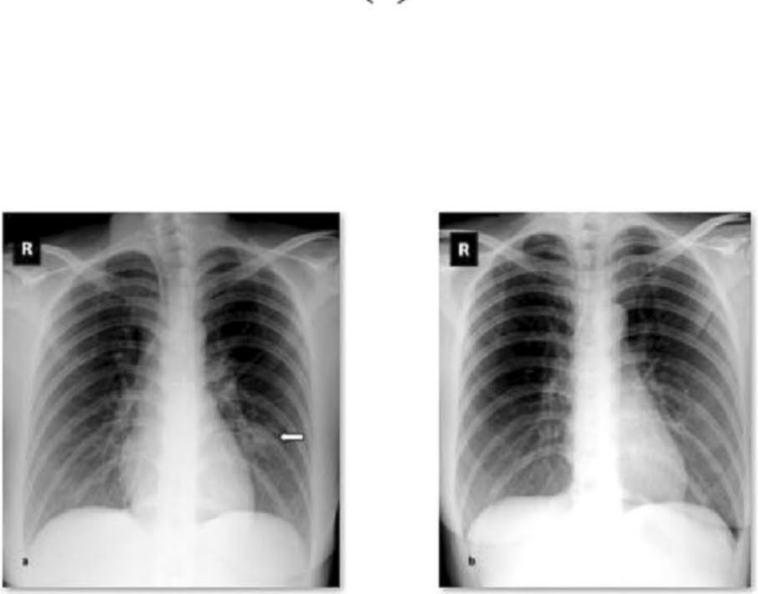

(b)

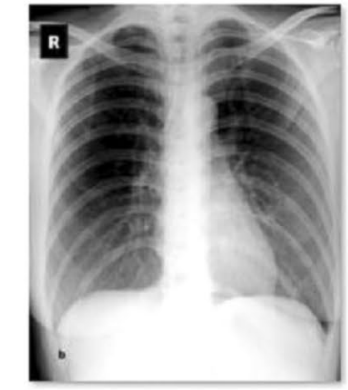

(b)

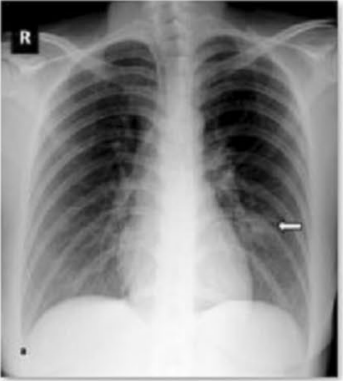

Fig. 6 Sample multi-class images

Table 1 Results analysis of FM-ANN technique on binary class

\begin{tabular}{lllll}
\hline Cross-validation & Sensitivity & Specificity & Accuracy & $F$ score \\
\hline fold 1 & 94.38 & 95.30 & 94.29 & 95.09 \\
Fold 2 & 95.17 & 95.43 & 95.64 & 94.85 \\
Fold 3 & 94.87 & 96.76 & 96.40 & 95.32 \\
Fold 4 & 94.32 & 95.89 & 95.88 & 96.83 \\
Fold 5 & 96.74 & 96.48 & 97.36 & 94.35 \\
Average & 95.10 & 95.97 & 95.91 & 95.29
\end{tabular}

provided maximum sensitivity of $94.72 \%$, specificity of $95.11 \%$, the accuracy of $95.32 \%$, and an $F$ score of $94.20 \%$ correspondingly. Likewise, under the CV-2, the developed FM-ANN technology has shown maximum sensitivity of $95.91 \%$, specificity of $95.73 \%$, accuracy of $94.34 \%$, and an $F$ score of $95.63 \%$, respectively.

Followed by, under the CV-3, the implied FM-ANN technique has offered greater sensitivity of $96.73 \%$, specificity of $96.46 \%$, accuracy of $96.70 \%$, and $F$ score of $94.09 \%$, respectively. Moreover, under the CV-4, the presented FM-ANN model has depicted high sensitivity of $94.98 \%$, specificity of $95.85 \%$, accuracy of $96.74 \%$,

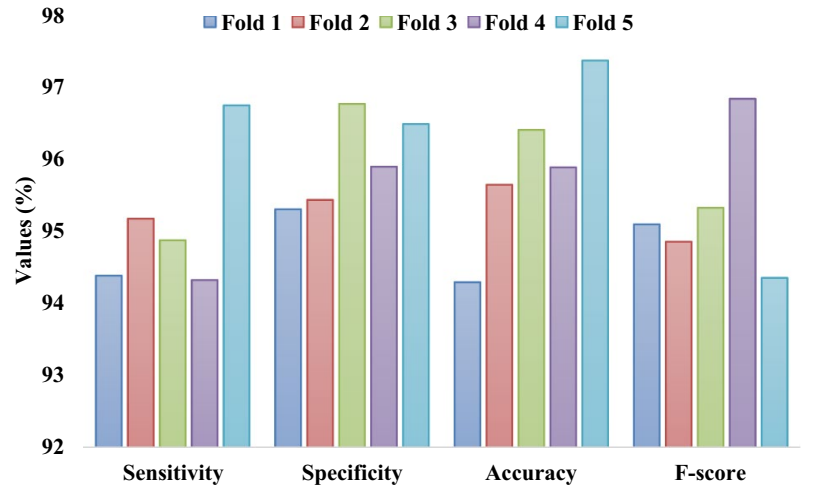

Fig. 7 Binary class analysis of FM-ANN model

and $F$ score of $95.87 \%$, respectively. Furthermore, under the CV-5, the projected FM-ANN approach has offered maximum sensitivity of $95.90 \%$, specificity of $96.54 \%$, accuracy of $95.38 \%$, and $F$ score of $96.97 \%$, respectively. In line with this, the $\mathrm{CV}$ average analysis of deployed FMANN technology has generated a sensitivity of $95.65 \%$, specificity of $95.94 \%$, accuracy of $95.70 \%$, and an $F$ score of $95.35 \%$ correspondingly. 
Table 2 Results analysis of FM-ANN technique on multi-class

\begin{tabular}{lllll}
\hline Cross-validation & Sensitivity & Specificity & Accuracy & $F$ score \\
\hline Fold 1 & 94.72 & 95.11 & 95.32 & 94.20 \\
Fold 2 & 95.91 & 95.73 & 94.34 & 95.63 \\
Fold 3 & 96.73 & 96.46 & 96.70 & 94.09 \\
Fold 4 & 94.98 & 95.85 & 96.74 & 95.87 \\
Fold 5 & 95.90 & 96.54 & 95.38 & 96.97 \\
Average & 95.65 & 95.94 & 95.70 & 95.35 \\
\hline
\end{tabular}

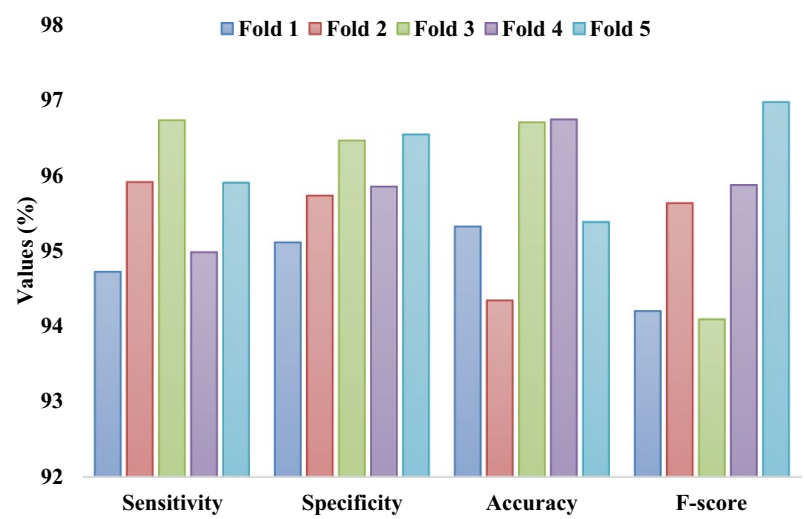

Fig. 8 Multi-class analysis of FM-ANN model

Figure 9 examines the classification performance of the FM-ANN model with existing methods in terms of sensitivity. It is noted that the DT and CNN models have led to the lowest sensitivity values of $87 \%$ and $87.73 \%$, respectively. Besides, it is noticed that the ANFIS and KNN models have resulted in slightly higher and closer sensitivity values of
$88.48 \%$ and $89 \%$. Similarly, the DTL and CoroNet models have appeared to even better classifiers with a sensitivity of $89.61 \%$ and $90 \%$, respectively.

On continuing with, the XGBoost, RNN and CNNLSTM models have reached to certainly higher $92 \%, 92.04 \%$, and $92.14 \%$, respectively. Along with that, the MLP and LR models have obtained an identical sensitivity value of 93\%. The LSTM and ANN models have reached moderate sensitivity values of $93.42 \%$ and $93.78 \%$. Next, the CNNRNN model has shown a somewhat acceptable sensitivity of 94.23\%. At last, the proposed FM-ANN model has achieved outstanding results by offering maximum sensitivity of $95.1 \%$ and $95.65 \%$ on binary and multiple classes, respectively. The figure investigates the classification function of the FM-ANN method with respect to specificity. It is clear that the CNN and MLP methodologies resulted in lower specificity values of $86.97 \%$ and $87.23 \%$ correspondingly. On the other hand, the ANFIS and DT approaches have provided better and nearby specificity values of $87.74 \%$ and 88.93\%. Along with that, the LR and XGBoost frameworks have showcased considerable classifiers with the specificity of $90.34 \%$ and $90.44 \%$ correspondingly. On continuing with, the KNN, RNN and ANN schemes have accomplished better $90.65 \%, 90.87 \%$ and $91.76 \%$, respectively. Likewise, the CNNLSTM and DTL approaches have attained best results with specificity values of $91.98 \%$ and $92.03 \%$, respectively. Then, the CoroNet model has showcased considerable specificity of $92.14 \%$. Next, the LSTM and CNNRNN frameworks have accomplished gradual specificity values of $92.64 \%$ and $92.67 \%$. Eventually, the projected FM-ANN method has reached optimal results by providing higher specificity of $95.97 \%$ and $95.94 \%$ on binary and several classes correspondingly.
Fig. 9 Comparative analysis of the proposed model in terms of sensitivity and specificity

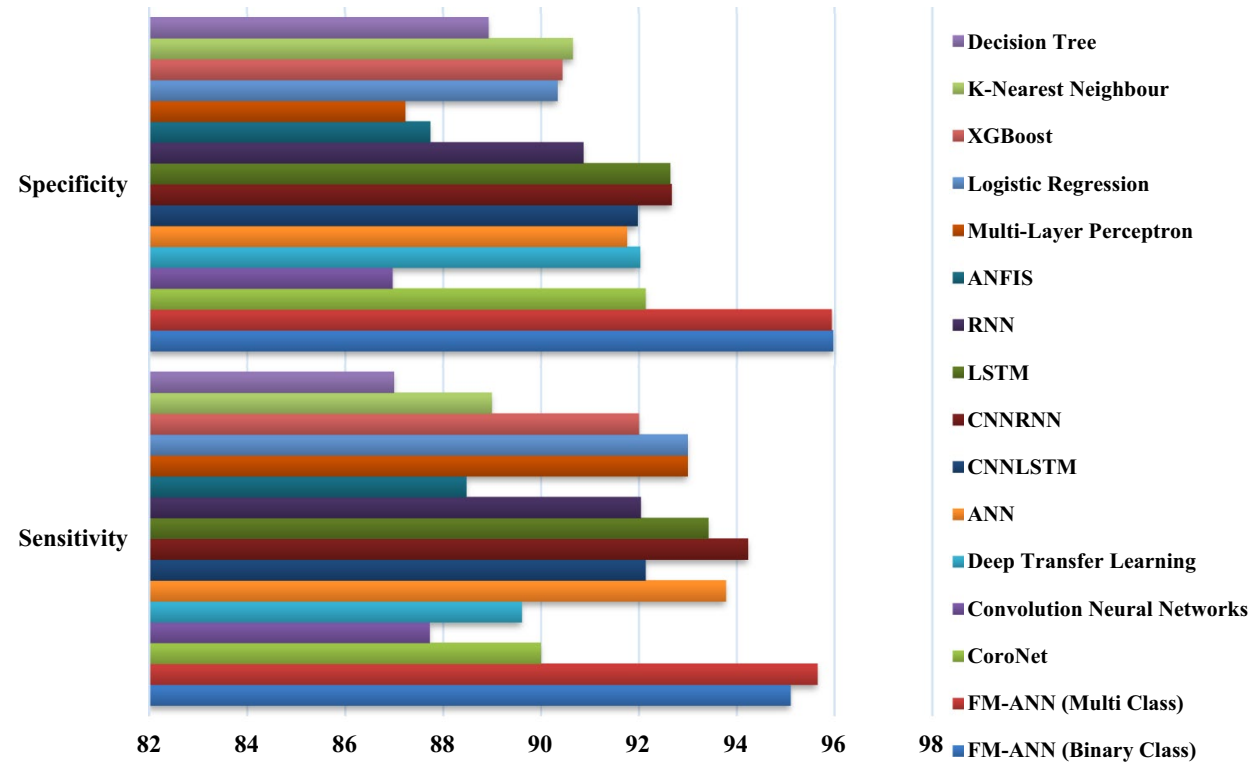


Figure 10 investigates the FM-ANN method's classification function with respect to accuracy. It is pointed out that the CNNLSTM and RNN approaches have resulted in the minimum and identical accuracy value of $84.16 \%$. Followed by, it is clear that the CNNRNN and ANN methodologies have provided moderate and closer accuracy values of $85.66 \%$ and $86 \%$. Likewise, the LSTM and DT frameworks have depicted even better classifiers with $86.66 \%$ and $86.71 \%$ accuracy. In line with this, the CNN, ANFIS, and KNN approaches have attained gradual $87.36 \%, 88.11 \%$ and $88.91 \%$, respectively. On continuing with, the CoroNet and DTL methodologies have accomplished considerable accuracy values of $90.21 \%$ and $90.75 \%$, respectively. Besides, the XGBoost and LR technologies have attained reasonable accuracy values of $91.57 \%$ and $92.12 \%$. Then, the MLP technology has illustrated better accuracy of $93.13 \%$. Consequently, the presented FM-ANN approach has attained maximum results by providing a higher accuracy of $95.91 \%$ and $95.70 \%$ on binary and multiple classes.

The figure investigates the classification function of the FM-ANN technique using $F$ score. DT and KNN schemes have resulted in lower $F$ score values of $87 \%$ and $89 \%$. On the other side, it is apparent that the ANFIS and CNN methodologies have accomplished better and closer $F$ score values of $89.04 \%$ and $89.65 \%$. Likewise, the CNNLSTM and ANN frameworks have led to even better classifiers with the $F$ score of $90.01 \%$ and $90.43 \%$, respectively. In line with this, the RNN and CoroNet approaches have reached moderate results with $F$ score values of $90.61 \%$ and $91 \%$, respectively. Similarly, the CNNRNN, ANN, and LSTM frameworks have attained considerable $91.20 \%$, $91.34 \%$, and $91.89 \%$, respectively. Besides, the XGBoost and LR methodologies have accomplished a better and closer $F$ score value of $92 \%$. Followed by, the MLP method has showcased a better $F$ score of $93 \%$. Finally, the proposed FM-ANN technique has accomplished optimal results by providing a high $F$ score of $95.29 \%$ and $95.35 \%$ on binary and multiple classes correspondingly.

From the detailed experimental validation, it is ensured that the FM-ANN model has resulted in effective diagnostic performance over the compared methods by providing a maximum accuracy of $95.1 \%$ and $95.65 \%$ on binary and multiple classes. The improved diagnostic performance is due to the fusion of three feature extraction models and the application of the SSA for the parameter tuning of the ANN.

\section{Conclusion}

This paper has developed an efficient fusion model for intelligent COVID-19 diagnosis using chest X-ray images. Initially, the preprocessing of images takes place using WF technique. Subsequently, the fusion-based feature extraction process is carried out by incorporating GLCM, GLRM, and LBP. Afterward, the optimal feature subset is selected by SSA. Finally, ANN is applied as a classification process to classify the infected and healthy patients. The proposed model's performance has been assessed using the Chest X-ray image dataset. The experimental values verified that the proposed FM-ANN model had achieved outstanding results by offering a maximum accuracy of $95.1 \%$ and $95.65 \%$ on binary and multiple classes. As a part of the future scope, the performance can be further enhanced using segmentation techniques.
Fig. 10 Comparative analysis of the proposed model in terms of accuracy and $F$ score

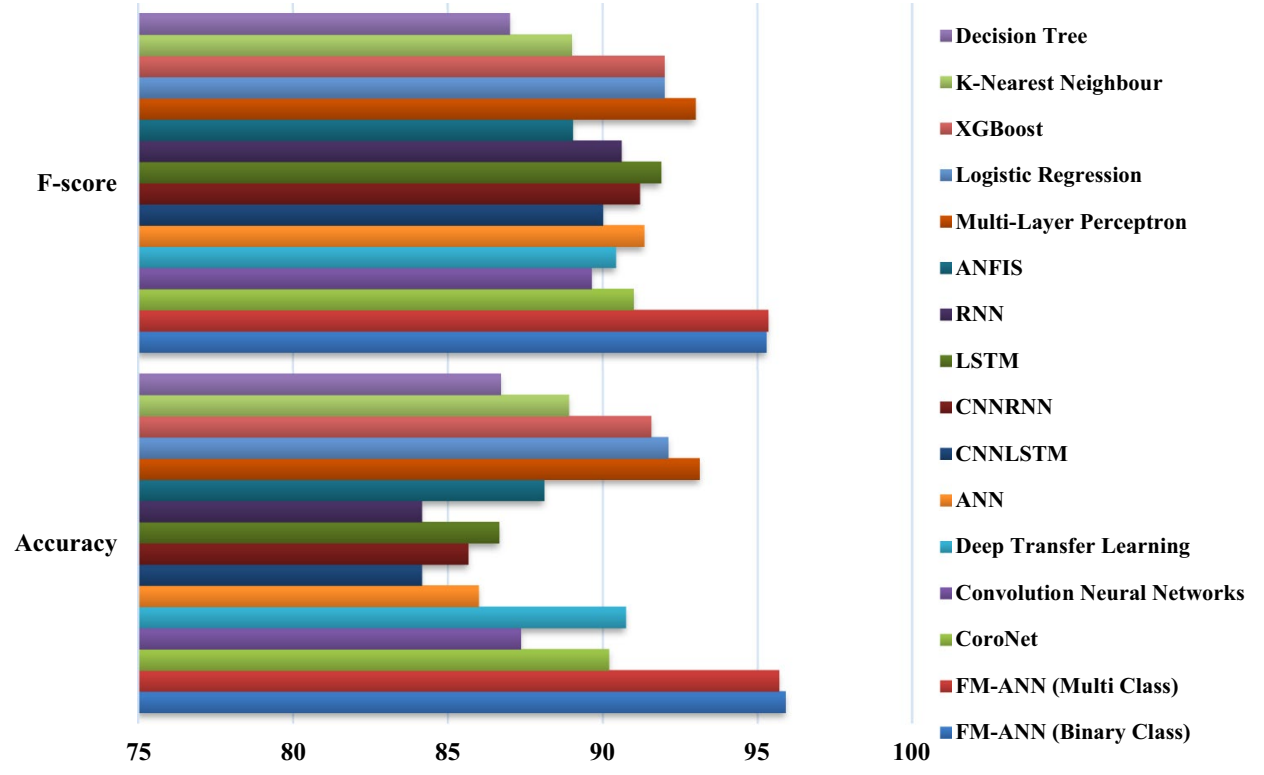


Acknowledgements Mohammad Shorfuzzaman sincerely acknowledge the financial support of Taif University Researchers Supporting Project Number (TURSP-2020/79), Taif University, Taif, Saudi Arabia.

\section{Declarations}

Conflict of interest The authors declare that they have no conflict of interest. The manuscript was written through contributions of all authors. All authors have given approval to the final version of the manuscript.

Ethical approval This article does not contain any studies with human participants or animal performed by any of the authors.

\section{References}

1. Dubey, A.K., Narang, S., Kumar, A., Sasubilli, S.M., Garcia-Diaz, V.: Performance estimation of machine learning algorithms in the factor analysis of COVID-19 dataset. CMC Comput. Mater. Continua 66(2), 1921-1936 (2021)

2. Saiz F.A., Barandiaran, I.: COVID-19 detection in chest X-ray images using a deep learning approach. Int. J. Interact. Multimed. Artif. Intell. 1 (2020) (in press)

3. Hossain, M.S.: Cloud-supported cyber-physical localization framework for patients monitoring. IEEE Syst. J. 11(1), 118-127 (2017)

4. Hossain, M.S., Muhammad, G., Alamri, A.: Smart healthcare monitoring: a voice pathology detection paradigm for smart cities. Multimedia Syst. 25(5), 565-575 (2019)

5. Muhammad, G., Hossain, M.S., Kumar, N.: EEG-based pathology detection for home health monitoring. IEEE J. Sel. Areas Commun. 39(2), 603-610 (2021)

6. Tiwari, P., Uprety, S., Dehdashti, S., Hossain, M.S.: TermInformer: unsupervised term mining and analysis in biomedical literature. Neural Comput. Appl. (2020). https://doi.org/10.1007/ s00521-020-05335-2

7. Tan, W., et al.: Multimodal medical image fusion algorithm in the era of big data. Neural Comput. Appl. 1-21 (2020)

8. Qian, J., et al.: A noble double-dictionary-based ECG compression technique for IoTH. IEEE Internet Things J. 7(10), 1016010170 (2020)

9. Hossain, M.S., Muhammad, G.: Deep learning based pathology detection for smart connected healthcares. IEEE Netw. 34(6), 120-125 (2020)

10. Min, W., et al.: Cross-platform multi-modal topic modeling for personalized inter-platform recommendation. IEEE Trans. Multimed. 17(10), 1787-1801 (2015)

11. Amin, S.U., et al.: Deep Learning for EEG motor imagery classification based on multi-layer CNNs feature fusion. Future Gener Comp Sy. 101, 542-554 (2019)

12. Hossain, M.S., Muhammad, G., Guizani, N.: Explainable AI and mass surveillance system-based healthcare framework to combat COVID-I9 like pandemics. IEEE Netw. 34(4), 126-132 (2020)

13 Abdulsalam, Y., Hossain, M.S.: COVID-19 networking demand: an auction-based mechanism for automated selection of edge computing services. IEEE Trans. Netw. Sci. Eng. (2020). https://doi. org/10.1109/TNSE.2020.3026637

14 Batista, A.F., Miraglia, J.L., Donato, T.H.R., Filho, A.D.P.C.: COVID-19 diagnosis prediction in emergency care patients: a machine learning approach. medRxiv (2020). https://doi.org/10. $1101 / 2020.04 .04 .20052092$
15. Schwab, P., Schütte, A.D., Dietz, B., Bauer, S.: predCOVID-19: a systematic study of clinical predictive models for coronavirus disease 2019. arXiv:2005.08302 (2020)

16. Pustokhin, D.A., Pustokhina, I.V., Dinh, P.N., Van Phan, S., Nguyen, G.N., Joshi, G.P., Shankar, K.: An effective deep residual network based class attention layer with bidirectional LSTM for diagnosis and classification of COVID-19. J. Appl. Stat. (2020). https://doi.org/10.1080/02664763.2020.1849057

17. Le, D.N., Parvathy, V.S., Gupta, D., et al.: IoT enabled depthwise separable convolution neural network with deep support vector machine for COVID-19 diagnosis and classification. Int. J. Mach. Learn. Cybern. (2021). https://doi.org/10.1007/ s13042-020-01248-7

18. Shankar, K., Perumal, E.: A novel hand-crafted with deep learning features based fusion model for COVID-19 diagnosis and classification using chest X-ray images. Complex Intell. Syst. (2020). https://doi.org/10.1007/s40747-020-00216-6

19 Jaiswal, A.K., et al.: Identifying pneumonia in chest X-rays: a deep learning approach. Measurement 145, 511-518 (2019)

20. Chouhan, V., et al.: A novel transfer learning based approach for pneumonia detection in chest X-ray images. Appl. Sci. 10(2), 559 (2020)

21. Rahman, M.A., et al.: B5G and explainable deep learning assisted healthcare vertical at the edge: COVID-I9 perspective. IEEE Netw. 34(4), 98-105 (2020)

22. Shorfuzzaman, M., Hossain, M.S.: MetaCOVID: A Siamese neural network framework with contrastive loss for n-shot diagnosis of COVID-19 patients. Pattern Recognit. 113, 107700 (2021)

23. Singh, G.A.P., Gupta, P.: Performance analysis of various machine learning-based approaches for detection and classification of lung cancer in humans. Neural ComputAppl 31(10), 6863-6877 (2019)

24. Esteva, A., Kuprel, B., Novoa, R.A., Ko, J., Swetter, S.M., Blau, H.M., et al.: Dermatologist-level classification of skin cancer with deep neural networks. Nature 542(7639), 115-118 (2017)

25. Liu, C., Cao, Y., Alcantara, M., Liu, B., Brunette, M., Peinado, J., et al.: Tx-cnn: detecting tuberculosis in chest X-ray images using convolutional neural network. In: 2017 IEEE International Conference on Image Processing (ICIP) , pp. 2314-2318. IEEE (2017)

26. Butt, C., Gill, J., Chun, D., Babu, B.A.: Deep learning system to screen coronavirus disease 2019 pneumonia. Appl. Intell. 6, 1122-1129 (2020)

27. Fanelli, D., Piazza, F.: Analysis and forecast of covid-19 spreading in China, Italy and France. Chaos SolitonsFract. 134, 109761 (2020)

28. Apostolopoulos, I.D., Mpesiana, T.A.: Covid-19: automatic detection from X-ray images utilizing transfer learning with convolutional neural networks. Phys. Eng. Sci. Med. 43, 635-640 (2020)

29. Li, L., Qin, L., Xu, Z., Yin, Y., Wang, X., Kong, B., et al.: Artificial intelligence distinguishes covid-19 from community acquired pneumonia on chest CT. Radiol. 296, 65-71 (2020)

30 Chimmula, V.K.R., Zhang, L.: Time series forecasting of covid-19 transmission in Canada using 1stm networks. Chaos SolitonsFract. (2020). https://doi.org/10.1016/j.chaos.2020.109864

31. Vallabhaneni, R.B., Rajesh, V.: Brain tumour detection using mean shift clustering and GLCM features with edge adaptive total variation denoising technique. Alex. Eng. J. 57(4), 2387-2392 (2018)

32. Raj, R.J.S., Shobana, S.J., Pustokhina, I.V., Pustokhin, D.A., Gupta, D., Shankar, K.: Optimal feature selection-based medical image classification using deep learning model in internet of medical things. IEEE Access 8, 58006-58017 (2020)

33. Khojastehnazhand, M., Ramezani, H.: Machine vision system for classification of bulk raisins using texture features. J. Food Eng. 271, 109864 (2020) 
34. Kaplan, K., Kaya, Y., Kuncan, M. and Ertunç, H.M.: Brain tumor classification using modified local binary patterns (LBP) feature extraction methods. Med. Hypotheses 139, 109696 (2020). https://doi.org/10.1016/j.mehy.2020.109696

35. Mirjalili, S., Gandomi, A.H., Mirjalili, S.Z., Saremi, S., Faris, H., Mirjalili, S.M.: Salp swarm algorithm: a bio-inspired optimizer for engineering design problems. Adv. Eng. Softw. 114, 163-191 (2017)

36. Hegazy, A.E., Makhlouf, M.A., El-Tawel, G.S.: Improved salp swarm algorithm for feature selection. J. King Saud Univ. Comput. Inf. Sci. 32(3), 335-344 (2020)

37. Devika, G., Ilayaraja, M., Shankar, K.: Optimal radial basis neural network (ORB-NN) For effective classification of clouds in satellite images with features. Int. J. Pure Appl. Math. 116(10), 309-329 (2017)

38. Gambhir, S., Malik, S.K., Kumar, Y.: PSO-ANN based diagnostic model for the early detection of dengue disease. New Horizons Transl. Med. 4(1-4), 1-8 (2017)

39. COVID-19 image data collection. https://www.github.com/ieee 8 023/covid-chestxray-dataset. Accessed 30 Mar 2020

Publisher's Note Springer Nature remains neutral with regard to jurisdictional claims in published maps and institutional affiliations.

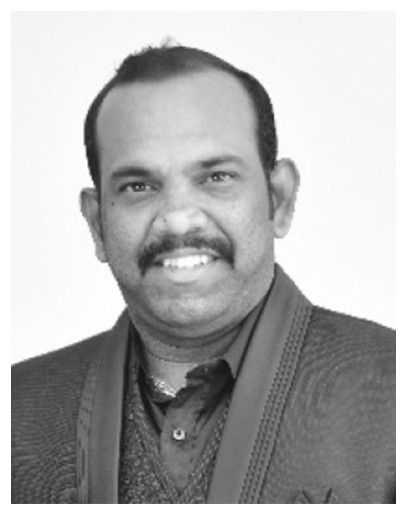

K. SHANKAR (Senior Member, IEEE) is currently a Postdoctoral Fellow with the Department of Computer Applications, Alagappa University, Karaikudi, India. He has authored/coauthored over 100+ ISI journal articles (with total Impact Factor $250+$ ) and more than 100 Scopus indexed articles. He has authored/edited conference proceedings, book chapters, and three books published by Springer. He has been a part of various seminars, paper presentations, research paper reviews, and convener and a session chair of the several conferences. He displayed vast success in continuously acquiring new knowledge and applying innovative pedagogies and has always aimed to be an effective educator and have a global outlook. His current research interests include healthcare applications, secret image sharing scheme, digital image security, cryptography, the Internet of Things, and optimization algorithms. He has guest-edited several special issues at many journals published by SAGE, TechScience, Inderscience, and MDPI. He has served as a Gust Editor and an Associate Editor in SCI, Scopus indexed journals, such as Elsevier, Springer, IGI, Wiley, \& MDPI. He has served as a chair (program, publications, technical committee, and track) on several international conferences. He has delivered several invited and keynote talks, and reviewed the technology leading articles for journals, such as Scientific Reports-Nature, the IEEE Transactions on Neural Networks and Learning Systems, IEEE Journal of
Biomedical and Health Informatics, IEEE Transactions on Reliability, the IEEE Access and the IEEE Internet of Things.

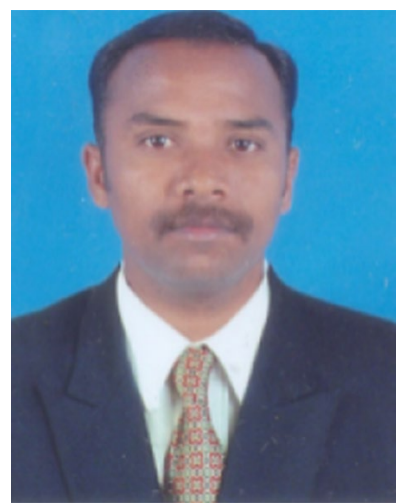

Eswaran Perumal received the M.Sc. degree in Computer Science and Information Technology from Madurai Kamaraj University, India in 2003, received M.Tech. and Ph.D. degree in Computer and Information Technology from Manonmaniam Sundaranar University, India in 2005 and 2010 respectively. In 2010, he joined the Department of Computer Science and Engineering, PSN College of Engineering and Technology, as Assistant Professor. Since May 2012, he has been with the Department of Computer Science and Engineering, Alagappa University, Karaikudi, India, as Assistant Professor. He was the recipient of the Junior Research Fellowship Award of the University Grants Commission, New Delhi in 2008. He has published more than 50 scientific papers in the field of Digital Image Processing and Data Mining. His research interests include Digital Image Processing, focusing on Color Image Edge Detection, Data Mining and Computer and Communication Network.

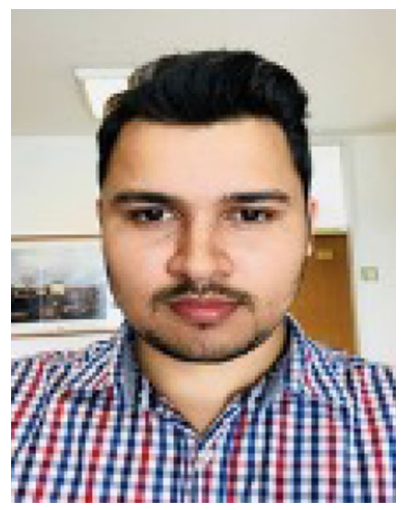

Prayag Tiwari is working as a Postdoctoral Researcher at the Aalto University. Previously, he was working as a Marie Curie Researcher and Ph.D. student at the University of Padova, Italy. He received his MS Degree from NUST MISIS, Moscow. He also worked as a Research Assistant at NUST MISIS, and he has had Teaching and Industrial work experience. He has several publications in top journals and conferences like CIKM, SIGIR, ICTIR, AAAI, ECIR, Applied Soft Computing, IEEE IoT, IEEE Access, Journal of Cleaner Production, Neural Computing and Application, etc. His research interests include Machine Learning, Deep Learning, Quantum-Inspired Machine Learning, Information Retrieval, and IoT.

Mohammad Shorfuzzaman is currently an Associate Professor with the Department of Computer Science, College of Computers and InformationTechnology (CCIT), Taif University, Taif, Saudi Arabia. He is also a member of the Big Data Analytics and Applications (BDAAG) Research Group,CCIT. His current research interests include applied artificial intelligence in the areas of computer vision, natural language processing, big data, andcloud computing. 


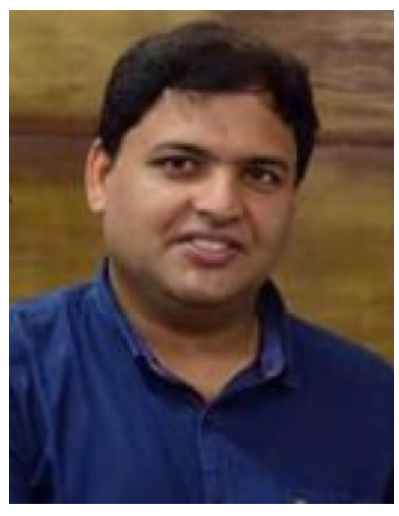

Deepak Gupta is an eminent academician; plays versatile roles and responsibilities juggling between lectures, research, publications, consultancy, community service, $\mathrm{PhD}$ and postdoctorate supervision etc. With 13 years of rich expertise in teaching and two years in industry; he focuses on rational and practical learning. He has contributed massive literature in the fields of Human-Computer Interaction, Intelligent Data Analysis, Nature-Inspired Computing, Machine Learning and Soft Computing. He is working as Assistant Professor at Maharaja Agrasen Institute of Technology (GGSIPU), Delhi, India. He has served as Editor-in-Chief, Guest Editor, Associate Editor in SCI and various other reputed journals (Elsevier, Springer, Wiley \& MDPI). He has actively been part of various reputed International conferences. He is not only backed with a strong profile but his innovative ideas, research's end-results and notion of implementation of technology in the medical field is by and large contributing to the society significantly. He is currently a Post-Doc researcher at University of Valladolid, Spain. He has completed his first Post-Doc from Inatel, Brazil, and Ph.D. from Dr. APJ Abdul Kalam Technical University. He has authored/Edited 50 books with National/International level publisher (Elsevier, Springer, Wiley, Katson). He has published 188 scientific research publications in reputed International Journals and Conferences including 99 SCI Indexed Journals of IEEE, Elsevier, Springer, Wiley and many more. $\mathrm{He}$ has also published 4 patents. He is the recipient of 2021 IEEE System Council Best Paper Award. He is Editor-in-Chief of OA Journal-Computers and Quantum Computing and Applications (QCAA), Associate Editor of Expert Systems (Wiley), Intelligent Decision Technologies (IOS Press), Journal of Computational and Theoretical Nenoscience, Honorary Editor of ICSES Transactions on Image Processing and Pattern Recognition. He is also a series editor of "Elsevier Biomedical Engineering" (Elsevier), "Intelligent Biomedical Data Analysis" (De Gruyter, Germany), "Explainable AI (XAI) for Engineering Applications" (CRC Press) and "Computational Intelligence for Data Analysis" (Bentham Science). He is appointed as Consulting Editor at Elsevier. $\mathrm{He}$ is also associated with various professional bodies like IEEE, ACM, ISTE, IAENG, IACSIT, SCIEI, ICSES, UACEE, Internet Society, SMEI, IAOP, and IAOIP. Invited as a Faculty Resource Person/Session Chair/Reviewer/TPC member in different FDP, conferences and journals. He is the convener of 'ICICC' conference series. Cumulative Impact Factor (CIF) is 309.96. 